\title{
OPTIMAL STOPPING OF TWO-PARAMETER PROCESSES ON NONSTANDARD PROBABILITY SPACES
}

\author{
ROBERT C. DALANG
}

\begin{abstract}
We prove the existence of optimal stopping points for upper semicontinuous two-parameter processes defined on filtered nonstandard (Loeb) probability spaces that satisfy a classical conditional independence hypothesis. The proof is obtained via a lifting theorem for elements of the convex set of randomized stopping points, which shows in particular that extremal elements of this set are ordinary stopping points.
\end{abstract}

\section{INTRODUCTION}

The optimal stopping problem for two-parameter processes has been the object of much research in recent years, starting with the fundamental paper [CG] of Cairoli and Gabriel. The discrete time version of the problem was then solved with increasing generality by Mandelbaum and Vanderbei [MV], Krengel and Sucheston [KS] and Mazziotto and Szpirglas [MS]. Several papers concerning the continuous time version of this problem have also appeared: Mazziotto [Ma] shows the existence of optimal stopping points for bi-Markov processes, and similar results are stated in [Mi] and [MM] for general two-parameter processes. However, the proofs contained in these two papers are not complete, and the question of existence of optimal stopping points for general two-parameter processes in continuous time is to be regarded as open (see Remark 7.4). However, in this paper, we shall prove the existence of optimal stopping points for upper semicontinuous two-parameter processes defined on a nonstandard (Loeb) probability space that satisfies the commutation property F4 of Cairoli and Walsh [CW].

The approach in this paper was motivated by the following considerations.

-The discrete time optimal stopping problem was well understood, but no continuous time extension had been obtained. In particular, no discretization argument seems feasible.

Received by the editors January 25, 1988.

1980 Mathematics Subject Classification (1985 Revision). Primary 60G40, 60G57, 60 G07.

Key words and phrases. Optimal stopping, two-parameter process, nonstandard analysis, randomized stopping point.

This research was accomplished while the author was completing his doctoral dissertation at the Ecole Polytechnique Fédérale de Lausanne, Switzerland. 
-Nonstandard probability theory, as developed by Loeb [L], Anderson [A], Keisler $[\mathrm{K}]$ and Hoover and Perkins [HP] provides a powerful tool for extending discrete case results to continuous time.

It thus seemed natural to study the optimal stopping problem via these methods, which have so far been little used in the general theory of two-parameter processes (the only case we are aware of is [MMe]).

Our main tool in this study of the optimal stopping problem is the notion of randomization. The convex compact set of randomized stopping times was first introduced in continuous time by Baxter and Chacon [BC], and used in the context of the single-parameter optimal stopping problem by Ghoussoub [G]: the property that makes this set useful is that extremal elements of the set of randomized stopping times are exactly ordinary stopping times. Now when trying to follow a similar procedure for two-parameter processes, one is hindered by the fact that the set $\mathscr{U}$ of randomized stopping points generally contains extremal elements which are not stopping points (a simple example is provided in [MM]). This fact turns out to be a consequence of the complex combinatorial structure of two-parameter filtrations (see [DTW]), and led Millet [Mi] and Mazziotto and Millet [MM] to try different randomizations.

As a matter of fact, the set of extremal elements of $\mathscr{U}$ seems to remain the set $\mathscr{T}$ of stopping points when the two-parameter filtration satisfies certain classical conditions, such as Hypothesis CQI of Krengel and Sucheston [KS] or Hypothesis F4 of Cairoli and Walsh [CW]. This was proved on finite probability spaces in [DTW] and on arbitrary complete probability spaces but in discrete time in [D2].

The main result of this paper is that the property $\mathscr{T}=\operatorname{ext} \mathscr{U}$ is again valid in continuous time, provided the underlying probability space is a nonstandard (Loeb) space. Existence of optimal stopping points for upper semicontinuous two-parameter processes is then obtained using a generalization of the regularity result for functionals of randomized stopping points obtained in [D1].

The use of nonstandard probability theory seems particularly natural due to the fact that the discrete time proof that $\mathscr{T}=\operatorname{ext} \mathscr{U}$ contained in [D2] relies on the construction of a particular optional increasing path $\left(Z_{n}\right)_{n \in \mathbb{N}}$ by a step by step procedure. In continuous time, one would imagine that a path $\left(Z_{u}\right)_{u \in \mathbf{R}_{+}}$with similar properties could be defined as the solution of a (random) differential equation of the form

$$
\frac{d Z_{u}}{d u}(\omega)=f\left(u,\left(Z_{v}\right)_{v \leq u}, \omega\right) .
$$

However, no regularity is to be expected from the function $f(\cdot, \cdot, \omega)$. Now certain stochastic differential equations with insufficiently regular coefficients are known not to have any (strong) solution (see Barlow [Ba]), and so it is improbable that $(*)$ would have a solution in any useful sense. On the other hand, Keisler [K] (Theorems 5.2 and 5.5) has shown under minimal regularity assumptions that stochastic differential equations have a (strong) solution 
when the probability space is hyperfinite, hence the use of these spaces in this paper. We feel that nonstandard probability theory may lead to solutions of several other problems in the theory of two-parameter processes, particularly in instances where the discrete case is solved, but the continuous time extension via classical methods does not seem to succeed.

\section{THE SET OF RANDOMIZED STOPPING POINTS}

Throughout this paper, we will primarily be concerned with stochastic processes indexed by $\mathbf{N}, \mathbf{D}_{n}$ or $\mathbf{R}_{+}$(single-parameter processes) or $\mathbf{N}^{2}, \mathbf{D}_{n}^{2}$ or $\mathbf{R}_{+}^{2}$ (two-parameter processes). Here $\mathbf{D}_{n}$ denotes the set of dyadic real numbers of order $n$. In the continuous case, we will often replace $\mathbb{R}_{+}$by $[0,1]$.

The letter $I$ (respectively $I^{2}$ ) will denote a single-parameter (respectively two-parameter) index set. The set $I$ is equipped with the usual total order, denoted $\leq$, whereas on $I^{2}$ it is natural to consider the two orders $\leq$ and $\Lambda$ defined by

$$
\begin{aligned}
& s=\left(s_{1}, s_{2}\right) \leq t=\left(t_{1}, t_{2}\right) \Leftrightarrow s_{1} \leq t_{1} \text { and } s_{2} \leq t_{2}, \\
& s=\left(s_{1}, s_{2}\right) \triangle t=\left(t_{1}, t_{2}\right) \Leftrightarrow s_{1} \leq t_{1} \text { and } s_{2} \geq t_{2} .
\end{aligned}
$$

We will use the notation $s<t$ to express that $s \leq t$ and $s \neq t$, whereas $s \wedge t$ will mean $s \Delta t$ and $s \neq t$, and $s \ll t$ will mean $s_{1}<t_{1}$ and $s_{2}<t_{2}$. Several kinds of intervals can be defined on $I^{2}:[s, t]=\left\{u \in I^{2}: s \leq u \leq t\right\}$, ]$s, t]=\left\{u \in I^{2}: s \ll u<t\right\}$ and so forth. In order to avoid introducing special symbols, we will set $] s, t]=\left\{u \in I^{2}: s<u \leq t\right\}$ when $s \leq t$ but $s_{1}=t_{1}$ or $s_{2}=t_{2}$.

In several instances, we will use the lexicographic (total) order $\leq_{1}$ on $I^{2}$ :

$$
s \leq_{1} t \Leftrightarrow\left(s_{1}<t_{1} \text { or }\left(s_{1}=t_{1} \text { and } s_{2} \leq t_{2}\right)\right) .
$$

The notation $s<_{1} t$ will mean $s \leq_{1} t$ and $s \neq t$.

We will often add to $I$ or $I^{2}$ an extra element, denoted in both cases $\infty$, and will set $\bar{I}=I \cup\{\infty\}, \bar{I}^{2}=I^{2} \cup\{\infty\}$. These sets will be equipped with their usual metric topologies, making them compact. We will also suppose that $t \leq \infty$, for all $t$ in either $I$ or $I^{2}$. The notations $\mathscr{B}(I), \mathscr{B}(\bar{I}), \mathscr{B}\left(I^{2}\right)$, $\mathscr{B}\left(\bar{I}^{2}\right)$ will denote in each case the Borel $\sigma$-algebra of the index set.

Let $(\Omega, \mathscr{F}, P)$ be a (complete) probability space. A two-parameter filtration is a family $\left(\mathscr{F}_{t}\right)_{t \in I^{2}}$ of sub- $\sigma$-algebras of $\mathscr{F}$ with the following properties.

F1. $\mathscr{F}_{0,0}$ contains all $P$-null sets;

F2. $s \leq t \Rightarrow \mathscr{F}_{s} \subset \mathscr{F}_{t}$;

F3. When $I=[0,1], \mathscr{F}_{s}=\bigcap_{t \in] s,(1,1)]} \mathscr{F}, \forall s \in I^{2}$. These properties are termed the "usual conditions" [DM, IV. 48].

Many results in the theory of two-parameter processes require a supplementary hypothesis on the two-parameter filtration, usually Hypothesis F4 of Cairoli and Walsh [CW]. 
F4. If $s, t, u \in I^{2}$ are such that $s \wedge t$ and $u=\left(s_{1}, t_{2}\right)$, then $\mathscr{F}_{s}$ is conditionally independent of $\mathscr{F}_{t}$ given $\mathscr{F}_{u}$.

This condition restricts the combinatorial complexity of the filtration (see [DTW, Theorems 3.6, 5.8 and 5.9]).

Associated with a two-parameter filtration is a set $\mathscr{T}$ of stopping points: a random variable $T: \Omega \rightarrow \bar{I}^{2}$ is a stopping point provided $\{T \leq t\} \in \mathscr{F}$, $\forall t \in I^{2}$.

Given a measurable real-valued process $X=\left(X_{t}\right)_{t \in \bar{I}^{2}}$, the optimal stopping problem is to determine a stopping point $T_{0}$ such that

$$
E\left(X_{T_{0}}\right)=\sup _{T \in \mathscr{T}} E\left(X_{T}\right)
$$

$T_{0}$ is then called optimal. We shall prove that optimal stopping points do exist on nonstandard filtered Loeb probability spaces that satisfy Hypothesis F4, under suitable regularity assumptions on the reward process $X$. This process may or may not be adapted (a process $\left(X_{t}\right)_{t \in I^{2}}$ is adapted to $\left(\mathscr{F}_{t}\right)_{t \in I^{2}}$ provided $X_{t}$ is $\mathscr{F}_{t}$-measurable, for all $t$ ).

The problem of existence of optimal stopping points reduces to the following: consider the map $\phi: \mathscr{T} \rightarrow \mathbf{R}$ defined by $T \mapsto \phi(T)=E\left(X_{T}\right)$, and show that this map attains its maximum on $\mathscr{T}$. It is thus natural to embed $\mathscr{T}$ into some larger "randomized" set $\mathscr{U}$ with certain convexity and compactness properties and on which $\phi$ can be extended to a function with sufficient regularity that a maximum over $\mathscr{U}$ will exist. The choice of randomization should be such that one can then recover a maximum in $\mathscr{T}$.

The regularity question for upper semicontinuous processes will be solved by a generalization of the result of [D1]. Furthermore, a natural way to randomize is to take the convex closure of $\mathscr{T}$ in an appropriate sense. This leads to the set of randomized stopping points, introduced by Baxter and Chacon [BC] in the single-parameter setting. The presentation of this set by Meyer [Me] and Ghoussoub [G] will be the most convenient for our purposes.

A randomized stopping point is a random probability measure $\mu(\omega, B), \omega \in$ $\Omega, B \in \mathscr{B}\left(\bar{I}^{2}\right)$ such that $\mu(\cdot,[0, t])$ is $\mathscr{F}_{t}$-measurable, for all $t$. Each stopping point $T$ identifies with the randomized stopping point $\mu_{T}$ defined by

$$
\mu_{T}(\omega, B)=I_{\{T \in B\}}(\omega), \quad \omega \in \Omega, B \in \mathscr{B}\left(\bar{I}^{2}\right),
$$

so $\mathscr{T}$ is "contained" in $\mathscr{U}$.

Let $\mathscr{C}$ denote the set of continuous real-valued processes $\left(X_{t}\right)_{t \in \bar{I}^{2}}$ such that $E\left(\sup _{t \in \bar{I}^{2}}|X|_{t}\right)<+\infty . \mathscr{C}$ equipped with the norm $\|X\|=E\left(\sup _{t \in \bar{I}^{2}}\left|X_{t}\right|\right)$ is a Banach space. It is well known that $\mathscr{U}$ is a subset of the unit ball in the dual $\mathscr{C}^{*}$ of $\mathscr{C}$ that is compact in the weak topology $\sigma\left(\mathscr{C}^{*}, \mathscr{C}\right)$ (see $[\mathrm{Me}, \mathrm{G}]$ ).

Furthermore, for $\left(X_{t}\right)_{t \in \bar{I}^{2}} \in \mathscr{C}$, the map $\Phi: \mathscr{U} \rightarrow \mathbf{R}$, defined by

$$
\Phi(\mu)=E\left(\int_{\bar{I}^{2}} X_{t}(\cdot) \mu(\cdot, d t)\right)
$$


is continuous on $\mathscr{U}$ and is an extension of $T \mapsto E\left(X_{T}\right)$. Hence, the existence of an optimal randomized stopping point is clear in this case. Now since $\Phi$ is affine and $\mathscr{U}$ is convex, $\Phi$ attains its maximum at an extremal element of $\mathscr{U}$. Thus we will have shown the existence of an optimal stopping point provided $\mathscr{T}=\operatorname{ext} \mathscr{U}$. This method was in fact used by Ghoussoub [G] for continuous single-parameter processes.

Now for two-parameter processes, it is clear that $\mathscr{T} \subset$ ext $\mathscr{U}$, but as mentioned in the introduction, the converse inclusion is false in general. Our purpose here is to show that the property $\mathscr{T}=$ ext $\mathscr{U}$ also holds in continuous time when $\Omega$ is a nonstandard (Loeb) space and the two-parameter filtration satisfies Hypothesis F4.

To see why this extension is feasible, let us first look at the set $\mathscr{U}$ when $I=\mathbf{N}$. In this case, a randomized stopping point can be identified with a positive weight process $\left(a_{t}\right)_{t \in \bar{N}^{2}}$ defined by $a_{t}(\omega)=\mu(\omega,\{t\})$ (i.e. $a_{t}$ is the random weight of $t$ for $\mu$ ). This weight process satisfies the following conditions:

(2.1) $a_{t} \geq 0$ a.s.;

(2.2) $a_{t}$ is $\mathscr{F}_{t}$-measurable, $\forall t \in \mathbb{N}^{2}$;

(2.3) $\sum_{t \in \bar{N}^{2}} a_{t}=1$ a.s.

These three properties characterize weight processes that correspond to randomized stopping points. The weights $\left(a_{t}\right)_{t \in \bar{N}^{2}}$ are very convenient to work with, and this was exploited in [D2]. Now when $I=\mathbf{R}_{+}^{2}$, a randomized stopping point can only be identified with a right-continuous nonnegative adapted process $\left(A_{t}\right)_{t \in \overline{\mathbf{R}}_{+}^{2}}$ such that $A_{\infty}=1$ a.s. and $\Delta_{\mathrm{j} s, t]} A \geq 0$ a.s., where

$$
\Delta_{\mathrm{Js}, t]} A= \begin{cases}A_{t}-A_{\left(s_{1}, t_{2}\right)}-A_{\left(t_{1}, s_{2}\right)}+A_{s} & \text { if } s \ll t, \\ A_{t}-A_{s} & \text { if } s \leq t \text { and } s_{1}=t_{1} \text { or } s_{2}=t_{2} .\end{cases}
$$

The main idea of this paper will be to "lift" a continuous time randomized stopping point to an (internal) weight process indexed by a hyperfinite set (the terminology from nonstandard probability theory will be recalled in §3). This weight process can then be manipulated as in the discrete case. Of course this procedure can only be carried out on a Loeb space and as mentioned in the introduction, it is not clear that a discretisation on a standard space can lead to a continuous time solution to the question of equality of $\mathscr{T}$ and ext $\mathscr{U}$. A corollary of this study will be a proof of the existence of optimal stopping points in continuous time.

Before introducing the nonstandard framework we will be working in, we recall the discrete case result of [D2]. For this, we need the notion of optional increasing path $[\mathrm{W}]$.

2.1. Definition. A family $Z=\left(Z_{u}\right)_{u \in \bar{I}}$ of stopping points is aptional increasing path (o.i.p.) provided $Z_{0} \equiv(0,0)$ a.s., $u \leq v \Rightarrow Z_{u} \leq Z_{v}$ a.s., and $\left|Z_{u}\right|=u$ a.s., $\forall u \in \bar{I}$ (for $t=\left(t_{1}, t_{2}\right),|t|$ denotes the sum $\left.t_{1}+t_{2}\right)$. If $I=\mathbf{D}_{n}$, 
we impose the supplementary condition $Z_{u+2^{-n}}$ is $\mathscr{F}_{Z_{u}}$-measurable, $\forall u \in \mathbf{D}_{n}$ (these o.i.p.'s are often called tactics: see [MV]).

Though the theorem below was proved under the weaker hypothesis CQI of Krengel and Sucheston [KS], we only need it for filtrations that satisfy Hypothesis F4.

2.2. Splitting Theorem. Let $(\Omega, \mathscr{F}, P)$ be a (complete) probability space, and $\left(\mathscr{F}_{t}\right)_{t \in \mathrm{N}^{2}}$ be a two-parameter filtration satisfying Hypothesis $\mathrm{F} 4$. Then:

(a) all extremal elements of the set of randomized stopping points are stopping points;

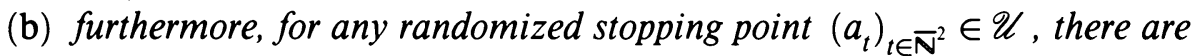
$\left(a_{t}^{1}\right)_{t \in \overline{\mathbf{N}}^{2}},\left(a_{t}^{2}\right)_{t \in \overline{\mathbf{N}}^{2}} \in \mathscr{U}$ and an o.i.p. $\left(Z_{n}\right)_{n \in \overline{\mathrm{N}}}$ such that:

(b1) $a_{t}=\frac{1}{2} a_{t}^{1}+\frac{1}{2} a_{t}^{2}$ a.s., $\forall t \in \overline{\mathbb{N}}^{2}$;

(b2) for almost all $\omega \in \Omega$,

$$
\begin{aligned}
& t \wedge Z_{|t|}(\omega) \Rightarrow a_{t}^{1}(\omega)=2 a_{t}(\omega), \quad a_{t}^{2}(\omega)=0, \\
& Z_{|t|}(\omega) \wedge t \Rightarrow a_{t}^{1}(\omega)=0, \quad a_{t}^{2}(\omega)=2 a_{t}(\omega) .
\end{aligned}
$$

(For a proof, see [D2, (4.22) and Theorem 4.23].)

In order to apply the Transfer Principle of Nonstandard Analysis (see (3.3)), we shall only need this result for index sets $I^{2}$ of the form $\left\{s \in \mathbb{N}^{2}: s \leq(n, n)\right\}$, for some $n \in \mathbb{N}$.

\section{PRELIMINARIES FROM NONSTANDARD PROBABILITY THEORY}

The nonstandard framework will be that of Keisler [K]: we work in an $\omega_{1}$ saturated enlargement $V\left({ }^{*} S\right)$ of a superstructure $V(S)$, where $S \supset \mathbb{R}$. The reader interested in familiarizing himself with the basics of nonstandard analysis should consult [HL]. The nonstandard theory of single-parameter stochastic processes is contained in [SB], and we follow their notation. In the hyperfinite setting, a comprehensive presentation with applications is given in [AFHL].

(3.1) Internal functions will generally be written $\tilde{f}, \tilde{g}$.

(3.2) The standard part of a finite element $r \in{ }^{*} \mathrm{R}$ is denoted st $(r)$. When $x, y \in{ }^{*} \mathrm{R}$ are such that $|x-y|<1 / n, \forall n \in \mathbb{N}$, we write $x \approx y$. If $s, t \in{ }^{*} \mathbb{R}^{2}$, $s \approx t$ means $s_{1} \approx t_{1}$ and $s_{2} \approx t_{2}$.

(3.3) Transfer Principle. Let $S_{1}, \ldots, S_{n} \in V(S)$. Any elementary statement which is true of $S_{1}, \ldots, S_{n}$ is true of ${ }^{*} S_{1}, \ldots,{ }^{*} S_{n}$.

(3.4) Countable Comprehension Principle. Let $X$ be an internal set, and $\left(x_{n}\right)_{n \in \mathrm{N}}$ be a sequence of elements of $X$. Then there exists an internal sequence $\left(y_{n}\right)_{n \in \mathbb{N}^{*}}$ of elements of $X$ such that $y_{n}=x_{n}, \forall n \in \mathbb{N}$.

(3.5) We fix $n_{0} \in{ }^{*} \mathbb{N} \backslash \mathbb{N}$, and set $L=n_{0} !, \Delta u=1 / L$. T denotes the internal set $\{0, \Delta u, 2 \Delta u, \ldots, 1\}$. Since $L$ is an infinite factorial, $\mathbf{T}$ contains all rational numbers in $[0,1]$. 
(3.6) If $(\Omega, \mathscr{A}, \bar{P})$ is an internal probability space, $(\Omega, L(\mathscr{A}), P)$ denotes the corresponding Loeb space, that is $L(\mathscr{A})$ is the (external) $\sigma$-algebra generated by $\mathscr{A}$, and $P$ is the unique $\sigma$-additive extension of $\operatorname{st}(\bar{P})$ to $L(\mathscr{A})$ [HP, $\S 3]$.

(3.7) An internal two-parameter filtration will be an internal family $\left(\mathscr{A}_{t}\right)_{t \in \mathbf{T}^{2}}$ of internal $*$-sub- $\sigma$-algebras of $\mathscr{A}$, such that

$$
s \leq t, s, t \in \mathrm{T}^{2} \Rightarrow \mathscr{A}_{s} \subset \mathscr{A}_{t} \text {. }
$$

This filtration is complete provided any internal subset $N$ of an internal set $M \in \mathscr{A}$ with $\bar{P}(M)=0$ belongs to $\mathscr{A}_{0,0}$. The standard part of $\left(\mathscr{A}_{t}\right)_{t \in \mathrm{T}^{2}}$ is the (ordinary) filtration $\left(\mathscr{F}_{t}\right)_{t \in[0,1]^{2}}$ defined by

$$
\mathscr{F}_{t}=\left(\bigcap_{\mathrm{st}(s) \gg t} \sigma\left(\mathscr{A}_{s}\right)\right) \vee \mathscr{N}, \quad t \in[0,1]^{2},
$$

where $\mathscr{N}$ denotes the family of null sets of $P$. It is easy to see that properties F1, F2 and F3 are satisfied.

(3.8) The family $\left(A_{t}\right)_{t \in \mathrm{T}^{2}}$ satisfies Hypothesis $\overline{\mathrm{F} 4}$ provided $s, t, u \in \mathbf{T}^{2}$, $s \underline{\Lambda} t, u=\left(s_{1}, t_{2}\right), B \in \mathscr{A}_{s}$, and $C \in \mathscr{A}_{t}$ imply

$$
\bar{P}\left(B \cap C \mid \mathscr{A}_{u}\right)=\bar{P}\left(B \mid \mathscr{A}_{u}\right) \bar{P}\left(C \mid \mathscr{A}_{u}\right) \text {. }
$$

(3.9) A lifting of a random variable $X$ defined on $(\Omega, L(\mathscr{A}), P)$ is an internal function $\tilde{X}: \Omega \rightarrow{ }^{*} \mathbf{R}$ which is $\mathscr{A}$-measurable (i.e. constant on atoms of $\mathscr{A})$, and such that $X=\operatorname{st}(\tilde{X}) \quad P$-a.s.

Throughout this paper, we will work on a fixed filtered Loeb space $(\Omega, \mathscr{F}=$ $L(\mathscr{A}), P,\left(\mathscr{F}_{t}\right)_{t \in[0,1]^{2}}$, where $P$ is the Loeb measure associated with an internal probability measure on $\mathscr{A}$, and $\left(\mathscr{F}_{t}\right)_{t \in[0,1]^{2}}$ is the standard part of an internal (complete) filtration $\left(\mathscr{A}_{t}\right)_{t \in \mathbf{T}^{2}}$.

3.1. Lemma. Fix $t \in[0,1]^{2}$. A random variable $X$ is $\mathscr{F}_{t}$-measurable provided $X$ has a lifting $\tilde{X}$ which satisfies the following condition:

$$
\text { there exists } s \in \mathbf{T}^{2}, s \approx t \text {, such that } \tilde{X} \text { is } \mathscr{A}_{s} \text {-measurable. }
$$

The proof of this lemma is omitted, as it is similar to the single-parameter case (see [HP, Theorem 3.2]).

3.2. Lemma. Let $X$ be a bounded random variable, and $\tilde{X}$ a bounded lifting of $X$. Fix $t \in[0,1]^{2}$. Then there is $u \approx t, u \in \mathbf{T}^{2}$ (depending on $X$ ) such that for $s \geq u, s \approx t, \bar{E}\left(\tilde{X} \mid \mathscr{A}_{s}\right)$ is a lifting of $E\left(X \mid \mathscr{F}_{t}\right)$.

Proof. By [HP, Lemma 3.3],

$$
\operatorname{st}\left(\bar{E}\left(\tilde{X} \mid \mathscr{A}_{s}\right)\right)=E\left(X \mid L\left(\mathscr{A}_{s}\right)\right) \text { a.s., } \forall s \in \mathbf{T}^{2} .
$$

Hence it is only necessary to prove that for some $u \in \mathrm{T}^{2}, u \approx t$,

$$
s \in \mathbf{T}^{2}, s \geq u, s \approx t \Rightarrow E\left(X \mid L\left(\mathscr{A}_{s}\right)\right)=E\left(X \mid \mathscr{F}_{t}\right) \text { a.s. }
$$


The proof of this statement is the straightforward two-parameter extension of the Remark following Lemma 8.4 in [Ke].

3.3. Proposition. Suppose $\left(A_{t}\right)_{t \in \mathrm{T}^{2}}$ satisfies Hypothesis $\overline{\mathrm{F} 4}$. Then $\left(\mathscr{F}_{t}\right)_{t \in[0,1]^{2}}$ satisfies Hypothesis $\mathrm{F} 4$.

Proof. Fix $s, t \in[0,1]^{2}$ such that $s \Delta t$, and set $u=\left(s_{1}, t_{2}\right)$. Let $B \in \mathscr{F}_{s}$, $C \in \mathscr{F}_{t}$. By Lemma 3.1, there are $\tilde{s}, \tilde{t} \in \mathrm{T}^{2}, \tilde{s} \approx s, \tilde{t} \approx t$, and internal sets $\widetilde{B} \in \mathscr{A}_{\hat{s}}, \widetilde{C} \in \mathscr{A}_{\hat{i}}$ such that $B=\widetilde{B}$ a.s. and $C=\widetilde{C}$ a.s. Using Lemma 3.2, we get for sufficiently large $\tilde{u} \approx u, \tilde{u} \in T^{2}$ :

$$
\begin{aligned}
P\left(B \cap C \mid \mathscr{F}_{u}\right) & =\operatorname{st}\left(\bar{P}\left(\widetilde{B} \cap \widetilde{C} \mid \mathscr{A}_{\tilde{u}}\right)\right) \\
& =\operatorname{st}\left(\bar{P}\left(\widetilde{B} \mid \mathscr{A}_{\tilde{u}}\right) \bar{P}\left(\widetilde{C} \mid \mathscr{A}_{\tilde{u}}\right)\right) \\
& =P\left(B \mid \mathscr{F}_{u}\right) P\left(C \mid \mathscr{F}_{u}\right) .
\end{aligned}
$$

The following proposition provides a canonical example of a filtered hyperfinite probability space which satisfies properties F1-F4.

3.4. Proposition. Let $\Omega=\Omega_{0}^{\mathrm{T}^{2}}$ be the (internal) set of all internal functions from $\mathbf{T}^{2}$ into some hyperfinite set $\Omega_{0}, \mathscr{A}$ be the algebra of internal sets in $\Omega$, and $\bar{P}$ the uniform counting measure on $\mathscr{A}$ (see $[\mathrm{Ke}, \S 1])$. For $t \in \mathrm{T}^{2}$, let $\mathscr{A}_{t}$ be the algebra of internal sets closed under the equivalence relation $\approx_{t}$ defined by

$$
\omega \approx_{t} \omega^{\prime} \Leftrightarrow \omega(s)=\omega^{\prime}(s), \forall s \leq t, s \in \mathbf{T}^{2} .
$$

Then $\left(\Omega, L(\mathscr{A}), P,(\mathscr{F})_{t \in[0,1]^{2}}\right)$ satisfies properties $\mathrm{F} 1, \mathrm{~F} 2, \mathrm{~F} 3$ and $\mathrm{F} 4$.

Proof. We only check Hypothesis F4. By Proposition 3.3, it is sufficient to check Hypothesis $\overline{\mathrm{F} 4}$ for $\left(A_{t}\right)_{t \in \mathbf{T}^{2}}$.

If $A$ is an internal set, let $|A|$ denote the internal cardinality of $A$, and let $\rho_{t}(\omega)$ denote the equivalence class of $\omega$ for $\approx_{t}$. Since each element of $\mathscr{A}_{t}$ is a hyperfinite union of disjoint equivalence classes $\rho_{t}(\omega)$, Hypothesis $\overline{\mathrm{F} 4}$ will hold provided for $s, t, u \in \mathrm{T}^{2}$ such that $s \underline{\Delta} t$ and $u=\left(s_{1}, t_{2}\right)$,

$$
\bar{P}\left(\rho_{s}\left(\omega^{\prime}\right) \cap \rho_{t}\left(\omega^{\prime \prime}\right) \mid \rho_{u}(\omega)\right)=\bar{P}\left(\rho_{s}\left(\omega^{\prime}\right) \mid \rho_{u}(\omega)\right) \bar{P}\left(\rho_{t}\left(\omega^{\prime \prime}\right) \mid \rho_{u}(\omega)\right),
$$

for all $\omega, \omega^{\prime}, \omega^{\prime \prime} \in \Omega$. Observe that both sides above are zero unless $\omega^{\prime} \approx_{u} \omega \approx_{u} \omega^{\prime \prime}$. In this case, the above equality is equivalent to

$$
\left|\rho_{s}\left(\omega^{\prime}\right) \cap \rho_{t}\left(\omega^{\prime \prime}\right)\right|=\left|\rho_{s}\left(\omega^{\prime}\right)\right|\left|\rho_{t}\left(\omega^{\prime \prime}\right)\right| / \mid \rho_{u}(\omega \mid .
$$

Since $\Omega=\Omega_{0}^{\mathbf{T}^{2}}$, the left-hand side of this equality is equal to

$$
\left|\Omega_{0}\right|^{L^{2}\left(\left(1-s_{2}\right)+\left(1-t_{1}\right) s_{2}+\left(s_{2}-t_{2}\right)\left(t_{1}-s_{1}\right)\right)}=\left|\Omega_{0}\right|^{L^{2}\left(1-s_{1} s_{2}-t_{1} t_{2}+s_{1} t_{2}\right)},
$$

where $L \in{ }^{*} \mathrm{~N}$ is defined in (3.5), and the right-hand side is equal to

$$
\left|\Omega_{0}\right|^{L^{2}\left(1-s_{1} s_{2}\right)}\left|\Omega_{0}\right|^{L^{2}\left(1-t_{1} t_{2}\right)} /\left|\Omega_{0}\right|^{L^{2}\left(1-u_{1} u_{2}\right)} .
$$


Since $u_{1} u_{2}=s_{1} t_{2}$, these two quantities are equal, completing the proof.

\section{The simultaneous lifting theOREM}

The first step towards obtaining a lifting theorem for continuous time randomized stopping points is to obtain such a theorem on a finite index set. This is no problem in the single-parameter case, but as will become apparent, it is quite nontrivial in the presence of two parameters.

Throughout the rest of this paper, we make the following assumption.

4.1. Assumption. The internal filtration $\left(\mathscr{A}_{t}\right)_{t \in[0,1]^{2}}$ satisfies Hypothesis $\overline{\mathrm{F} 4}$.

4.2. Simultaneous Lifting Theorem. Fix $n \in \mathbb{N}$, and set $I=\{0,1 / n$, $2 / n, \ldots, 1\}$. Let $\left(a_{t}\right)_{t \in I^{2}}$ be a family of real random variables such that

(4.1) $a_{t}$ is $\mathscr{F}_{t}$-measurable, $\forall t \in I^{2}$;

(4.2) $a_{t} \geq 0$ a.s., $\forall t \in I^{2}$;

(4.3) $\sum_{t \in I^{2}} a_{t}=1$ a.s.

Then there is a family $\left(\tilde{a}_{t}\right)_{t \in I^{2}}$ of internal functions from $\Omega$ into ${ }^{*} \mathbf{R}$ such that

(4.4) $\operatorname{st}\left(\tilde{a}_{t}\right)=a_{t}$ a.s.;

(4.5) for each $t \in I^{2}$, there is $s \in \mathrm{T}^{2}, s \approx t$ such that $\tilde{a}_{t}$ is $\mathscr{A}_{s}$-measurable;

(4.6) $\tilde{a}_{t}(\omega) \geq 0, \forall \omega \in \Omega, \forall t \in I^{2}$;

(4.7) $\sum_{t \in I^{2}} \tilde{a}_{t}(\omega)=1, \forall \omega \in \Omega$.

4.3. Remark. The difficult point in this theorem is to replace the (external) "a.s." relationships in (4.2) and (4.3) by the internal relations (4.6) and (4.7) valid for each $\omega \in \Omega$. Though the proof seems nontrivial already for $n=2$, and uses the conditional supremum operator introduced in [D2], its proof would be quite straightforward in the single-parameter case, when $I^{2}$ is replaced by $I$. We briefly indicate how the theorem could be proved in this case.

Let $\tilde{b}_{t}$ be a lifting of $\sum_{s \leq t} a_{s}$, such that $0 \leq \tilde{b}_{t}(\omega) \leq 1, \forall \omega \in \Omega$, and for some $s^{t} \approx t, \tilde{b}_{t}$ is $\mathscr{A}_{s^{t}}$-measurable. Set $\tilde{c}_{t}=\sup _{s \leq t} \tilde{b}_{s}, \tilde{a}_{0}=\tilde{c}_{0}$, and

$$
\begin{aligned}
& \tilde{a}_{t}=\tilde{c}_{t}-\tilde{c}_{t-1 / n}, \quad t \in I \backslash\{0,1\}, \\
& \tilde{a}_{1}=1-\tilde{c}_{(n-1) / n} .
\end{aligned}
$$

Then $\left(\tilde{a}_{t}\right)_{t \in I}$ has the desired properties.

Before proving Theorem 4.2, we recall the definition and main properties of the conditional supremum operator $S(Y \mid \mathscr{G})$ introduced in [D2]: given a sub$\sigma$-algebra $\mathscr{G}$ of $\mathscr{F}$, and a bounded random variable $Y, S(Y \mid \mathscr{G})$ is the $\mathscr{G}$ measurable random variable defined by $S(Y \mid \mathscr{G})=\operatorname{ess} \inf Z$, where the essential infimum is taken over all $Z \geq Y$ which are $\mathscr{G}$-measurable. $S(\cdot \mid \cdot)$ has the following properties, which we recall for ease of reference.

(4.8) $\mathscr{G}_{1} \subset \mathscr{G}_{2} \Rightarrow S\left(Y \mid \mathscr{G}_{2}\right) \leq S\left(Y \mid \mathscr{G}_{1}\right)$;

(4.9) If $X$ is $\mathscr{G}$-measurable, then $S(X+Y \mid \mathscr{G})=X+S(Y \mid \mathscr{G})$;

(4.10) If $\left(\mathscr{F}_{t}\right)_{t \in I^{2}}$ satisfies Hypothesis $\mathrm{F} 4$, and $s, t, u \in I^{2}$ are such that $s \Delta t, u=\left(s_{1}, t_{2}\right)$ and if $Y$ is $\mathscr{F}_{s}$-measurable, then $S\left(Y \mid \mathscr{F}_{t}\right)=S\left(Y \mid \mathscr{F}_{u}\right)$. 
((4.8) is clear; (4.9) follows from [D2, Lemma 4.7 (f)] and (4.10) follows from [D2, Proposition 4.12(a) and (b)].)

Proof of Theorem 4.2. For $t \in I^{2}$, set $R_{t}^{-}=\left\{s \in I^{2}: s \Delta t\right\}$, and $A_{t}^{-}=$ $\sum_{s \in R_{t}^{-}} a_{s}$. Observe that $0 \leq S\left(A_{t}^{-} \mid \mathscr{F}\right) \leq 1$ a.s., since $0 \leq A_{t}^{-} \leq 1$ a.s. Since $a_{t}$ and $S\left(A_{t}^{-} \mid \mathscr{F}_{t}\right)$ are $\mathscr{F}_{t}$-measurable, there exist by Lemma 3.1 two internal functions $\tilde{b}_{t}, \tilde{S}_{t}: \Omega \rightarrow{ }^{*}[0,1]$ such that

(4.11) $\operatorname{st}\left(\tilde{b}_{t}\right)=a_{t}$ a.s., $\operatorname{st}\left(\widetilde{S}_{t}\right)=S\left(A_{t}^{-} \mid \mathscr{F}_{t}\right)$ a.s.,

(4.12) for some $s^{t} \approx t, \tilde{b}_{t}$ and $\tilde{S}_{t}$ are $\mathscr{A}_{s^{t}}$-measurable.

We can now define $\tilde{a}_{t}, t \in I^{2}$, by induction in increasing order for $\leq_{1}$ (the lexicographic order on $I^{2}$ ). Throughout this proof, $k$ and $l$ will denote elements of $I$. Set

$$
\tilde{a}_{0,0}=\min \left(\tilde{b}_{0,0}, \tilde{S}_{0,0}\right)
$$

and suppose by induction that $\tilde{a}_{s}$ has been defined, for $s<_{1} t$. Then set

$$
\tilde{a}_{t}=\max \left(0, \min \left(\tilde{b}_{t}, \min _{\substack{0 \leq k<t_{1} \\ 0 \leq l \leq t_{2}}}\left(\tilde{S}_{t_{1}, l}-\widetilde{S}_{k, t_{2}}-\sum_{\substack{u<t \\ u \in R_{t_{1}, l} \backslash R_{k, l_{2}}^{-}}} \tilde{a}_{u}\right)\right)\right)
$$

if $t \neq(1,1)$, and

$$
\tilde{a}_{1,1}=1-\sum_{t \in I^{2} \backslash\{(1,1)\}} \tilde{a}_{t} .
$$

Then property (4.7) is trivially satisfied. Before showing that properties (4.4), (4.5) and (4.6) hold, we prove the following lemmas.

4.4. Lemma. Fix $t \in I^{2}$, and $0 \leq k<t_{1}, 0 \leq l \leq t_{2}$. Then

$$
a_{t} \leq S\left(A_{t_{1}, l}^{-} \mid \mathscr{F}_{t_{1}, l}\right)-S\left(A_{k, t_{2}}^{-} \mid \mathscr{F}_{k, t_{2}}\right)-\sum_{\substack{u<t \\ u \in R_{t_{1},}^{-} \backslash R_{k, t_{2}}^{-}}} a_{u} \text { a.s. }
$$

Proof. Since $(\mathscr{F})_{t \in I^{2}}$ satisfies Hypothesis F4, (4.10) implies that

$$
S\left(A_{k, t_{2}}^{-} \mid \mathscr{F}_{k, t_{2}}\right)+\sum_{\substack{u \leq t \\ u \in R_{t_{1},}^{-} \backslash R_{k, t_{2}}^{-}}} a_{u}=S\left(A_{k, t_{2}}^{-} \mid \mathscr{F}_{t}\right)+\sum_{\substack{u \leq t \\ u \in R_{t_{1},}^{-}, \backslash R_{k, t_{2}}^{-}}} a_{u},
$$

which, by (4.9), is equal to

$$
S\left(A_{k, t_{2}}^{-}+\sum_{\substack{u \leq t \\ u \in R_{t_{1}, l}^{-} \backslash R_{k, t_{2}}^{-}}} a_{u} \mid \mathscr{F}_{t}\right) \leq S\left(A_{t_{1}, l}^{-} \mid \mathscr{F}_{t}\right)
$$

By (4.8), this is not greater than $S\left(A_{t_{1}, l}^{-} \mid \mathscr{F}_{t_{1}, l}\right)$. This clearly implies the statement of the lemma. 
4.5. Lemma. Fix $t_{1}, l \in I$, and $\omega \in \Omega$, and suppose $\tilde{a}_{t_{1}, t_{2}}(\omega)>0$, for some $t_{2} \geq l$ with $\left(t_{1}, t_{2}\right) \neq(1,1)$. Then

$$
\sum_{\substack{s \in R_{t_{1}, l}^{-} \\ s \neq(1,1)}} \tilde{a}_{s}(\omega) \leq \widetilde{S}_{t_{1}, l}(\omega)
$$

Proof. We first show that the statement of the lemma holds when $t_{1}=0$. Suppose $\tilde{a}_{0, t_{2}}(\omega)>0$ for some $t_{2} \geq l$. Let $t_{2} \in I$ be maximal with this property. Then

$$
\sum_{s \in R_{0, l}^{-}} \tilde{a}_{s}(\omega)=\sum_{l \leq s_{2} \leq t_{2}} \tilde{a}_{0, s_{2}}(\omega) .
$$

Now by (4.13), $\tilde{a}_{0, t_{2}}(\omega)>0$ implies

$$
\tilde{a}_{0, t_{2}}(\omega) \leq \widetilde{S}_{0, l}(\omega)-\sum_{l \leq s_{2}<t_{2}} \tilde{a}_{0, s_{2}}(\omega)
$$

and thus

$$
\sum_{l \leq s_{2} \leq t_{2}} \tilde{a}_{0, s_{2}}(\omega) \leq \tilde{S}_{0, l}(\omega) \text {. }
$$

By (4.14), the lemma holds for $t_{1}=0$.

Suppose now by induction that the statement of the lemma holds for $0 \leq$ $t_{1}^{\prime}<t_{1}$, and show that it holds for $t_{1}$. Fix $l \in I$, and suppose $\tilde{a}_{t_{1}, t_{2}}(\omega)>0$, for some $t_{2} \geq l$, with $\left(t_{1}, t_{2}\right) \neq(1,1)$. Let $t_{2}$ be maximal with this property. Case 1. $\tilde{a}_{t_{1}^{\prime}, t_{2}^{\prime}}(\omega)=0, \forall t_{1}^{\prime}<t_{1}, t_{2}^{\prime} \geq t_{2}$. Then

$$
\sum_{\substack{s \in R_{t_{1}, l}^{-} \\ s \neq(1,1)}} \tilde{a}_{s}(\omega)=\sum_{\substack{s \leq\left(t_{1}, t_{2}\right) \\ s \in R_{t_{1}, .}^{-} \backslash R_{t_{1}-1, t_{2}}^{-}}} \tilde{a}_{s}(\omega)=\tilde{a}_{t_{1}, t_{2}}(\omega)+\sum_{\substack{s<\left(t_{1}, t_{2}\right) \\ s \in R_{t_{1},}^{-}, \backslash R_{t_{1}-1, t_{2}}^{-}}} \tilde{a}_{s}(\omega) .
$$

By (4.13), $\tilde{a}_{t_{1}, t_{2}}(\omega)>0$ implies that the last expression above is not greater than

$$
\widetilde{S}_{t_{1}, l}(\omega)-\widetilde{S}_{t_{1}-1, t_{2}}(\omega) \leq \widetilde{S}_{t_{1}, l}(\omega)
$$

which implies the desired property.

Case 2. For some $k<t_{1}$ and $t_{2}^{\prime} \geq t_{2}, \tilde{a}_{k, t_{2}^{\prime}}(\omega)>0$. Let $k$ be maximal with this property. Then

$$
\sum_{s \in R_{t_{1}, l}^{-}} \tilde{a}_{s}(\omega)=\sum_{s \in R_{k . / 2}^{-}} \tilde{a}_{s}(\omega)+\sum_{\substack{s<\left(t_{1}, t_{2}\right) \\ s \in R_{t_{1}, .}^{-} \backslash R_{k, t_{2}}^{-}}} \tilde{a}_{s}(\omega)+\tilde{a}_{t_{1}, t_{2}}(\omega)
$$

Applying the induction hypothesis to the first term on the right-hand side of (4.15) and using the fact that $\tilde{a}_{t_{1}, t_{2}}(\omega)>0$, we see by (4.13) that this last expression is not greater than

$$
\widetilde{S}_{k, t_{2}}(\omega)+\widetilde{S}_{t_{1}, l}(\omega)-\widetilde{S}_{k, t_{2}}(\omega)=\widetilde{S}_{t_{1}, l}(\omega)
$$


This completes the proof of the lemma.

End of the proof of Theorem 4.2. To see (4.4), we proceed by induction in increasing order for $\leq_{1}$. Use Lemma 4.4 and (4.11) and (4.13) to see that

$$
\begin{aligned}
\operatorname{st}\left(\tilde{a}_{t}\right) & =\max \left(0, \min \left(\operatorname{st}\left(\tilde{b}_{t}\right), \min _{\substack{0 \leq k<t_{1} \\
0 \leq l \leq t_{2}}}\left(\operatorname{st}\left(\tilde{S}_{t_{1}, l}-\tilde{S}_{k, t_{2}}-\sum_{\substack{u<t \\
u \in R_{t_{1}, l}^{-} \backslash R_{k, t_{2}}^{-}}} \tilde{a}_{u}\right)\right)\right)\right) \\
& =\operatorname{st}^{\prime}\left(\tilde{b}_{t}\right)=a_{t} \text { a.s. }
\end{aligned}
$$

Again proceeding by induction in increasing order for $\leq_{1}$, we see that (4.5) is implied by (4.12) and (4.13). Now (4.6) clearly holds for all $t \in I^{2} \backslash\{(1,1)\}$ by (4.13). To see that $(4.6)$ holds for $t=(1,1)$, we must show that

$$
\sum_{s<(1,1)} \tilde{a}_{s}(\omega) \leq 1, \quad \forall \omega \in \Omega
$$

Let $t \in I^{2} \backslash\{(1,1)\}$ be $\leq_{1}$-maximal such that $\tilde{a}_{t}(\omega)>0$. Using Lemma 4.5, we see that

$$
\sum_{s<(1,1)} \tilde{a}_{s}(\omega)=\sum_{s \in R_{t_{1}, 0}^{-} \backslash\{(1,1)\}} \tilde{a}_{s}(\omega) \leq \widetilde{S}_{t_{1}, 0}(\omega) \leq 1 .
$$

This concludes the proof of the theorem.

\section{A LifTING THEOREM AND A PROJECTION THEOREM FOR RANDOMIZED STOPPING POINTS}

5.1. Definition. An internal weight process on $\mathrm{T}^{2}$ is an internal function $\delta \alpha: \Omega$ $\times \mathbf{T}^{2} \rightarrow{ }^{*}[0,1]$. Such a weight process defines a random internal additive measure $\bar{\alpha}$ on the internal algebra of internal subsets of $T^{2}$ by the formula

$$
\bar{\alpha}(\omega, B)=\sum_{t \in B} \delta \alpha(\omega, t),
$$

where $\omega \in \Omega$ and $B$ is an internal subset of $\mathrm{T}^{2}$. If $\bar{\alpha}$ is finite a.s., the $\sigma$-additive extension of $\operatorname{st}(\bar{\alpha}(\omega, \cdot))$ to the Borel $\sigma$-algebra on $T^{2}$ is denoted $\alpha(\omega, \cdot)$ (the Borel $\sigma$-algebra is generated by the algebra of all internal subsets of $\left.\mathrm{T}^{2}\right)$.

The object of this section is to show how to lift a randomized stopping point to an internal weight process, and conversely, how to obtain a randomized stopping point from an internal weight process. Our method for lifting relies on the Simultaneous Lifting Theorem 4.1, and is quite different from the singleparameter lifting theorem of [SB, Chapter 7.1], which uses Skorohod's topology on right-continuous processes with left limits. Recall that Assumption 4.1 is in force. 
5.2. Lifting Theorem. Let $\left(A_{t}\right)_{t \in[0,1]^{2}}$ be a randomized stopping point. Then there is $h \in{ }^{*} \mathrm{~N} \backslash \mathbf{N}, h \leq n_{0} \quad\left(n_{0}\right.$ is defined in (3.5)), an internal weight process $\delta \alpha$, and a (generally external) $P$-null set $N \subset \Omega$ such that

(a) for each $t \in \mathrm{T}^{2}, \delta \alpha(\cdot, t)$ is $\mathscr{A}_{t+(1 / h, 1 / h)}$-measurable;

(b) $\left.\left.\Delta_{\mathrm{l}, t]} A(\omega)=\alpha\left(\omega,{ }^{*}\right] s, t\right] \cap \mathrm{T}^{2}\right), \forall s, t \in \mathrm{D}^{2}, s<t, \forall \omega \in \Omega \backslash N$;

(c) $\bar{\alpha}\left(\omega, \mathbf{T}^{2}\right)=1, \forall \omega \in \Omega$.

(D denotes the dyadics in $[0,1]$. Throughout this section we use the following convention: $\left.t+(1 / h, 1 / h)=\left(\min \left(t_{1}+1 / h, 1\right), \min \left(t_{2}+1 / h, 1\right)\right)\right)$.

Proof. Set $k=\left(k_{1}, k_{2}\right), k^{-}=\left(k_{1}-1, k_{2}-1\right), k^{+}=\left(k_{1}+1, k_{2}+1\right)$. Using Theorem 4.2, we see that for each $n \in \mathbb{N}$ and $0 \leq k_{1}, k_{2} \leq 2^{n}$, there is a $P$-null set $N_{\left(k_{1}, k_{2}\right)}^{n}$ and an internal function $\delta \alpha_{\left(k_{1}, k_{2}\right)}^{n}: \Omega \rightarrow{ }^{*}[0,1]$ such that

$$
\omega \in \Omega \backslash N_{k}^{n} \Rightarrow \operatorname{st}\left(\delta \alpha_{k}^{n}(\omega)\right)=\Delta_{\left.\left.2^{-n}\right] k^{-}, k\right]} A(\omega),
$$$$
\delta \alpha_{k}^{n} \text { is } \mathscr{A}_{2^{-n} k^{+}-\text {measurable, }}
$$

$$
\sum_{0 \leq k_{1}, k_{2} \leq 2^{n}} \delta \alpha_{\left(k_{1}, k_{2}\right)}^{n}(\omega)=1, \quad \forall \omega \in \Omega
$$

Let $B$ denote the set of internal functions from $\Omega \times \mathrm{T}^{2}$ into ${ }^{*}[0,1] . B$ is internal (see [HL, Ex. II.6.12]). For $n \in \mathbb{N}$, we define an element $\delta \alpha^{n}$ of $B$ by setting

$$
\delta \alpha^{n}(\omega, t)= \begin{cases}\delta \alpha_{\left(k_{1}, k_{2}\right)}^{n}(\omega) & \text { if } t=k 2^{-n}, \text { for some } 0 \leq k_{1}, k_{2} \leq 2^{n} \\ 0 & \text { otherwise. }\end{cases}
$$

Observe that by $(5.2)$,

$$
\left.\left.\delta \alpha^{n}(\cdot, t) \text { is } \mathscr{A}_{2^{-n} k^{+}} \text {-measurable, } \forall t \in \in^{*}\right] 2^{-n} k^{-}, 2^{-n} k\right] \cap \mathrm{T}^{2} .
$$

Set $\tilde{N}=\bigcup_{n \in N} \bigcup_{0 \leq k_{1}, k_{2} \leq 2^{n}} N_{\left(k_{1}, k_{2}\right)}^{n}$. Then there is a sequence $\left(N^{n}\right)_{n \in N}$ of internal subsets of $\Omega$ such that $N^{n} \supset \tilde{N}$ and

$$
\begin{aligned}
& \bar{P}\left(N^{n}\right)<1 / n \text { and } N^{m} \supset N^{n}, \quad \forall m \leq n, \\
& 0 \leq k_{1}, k_{2} \leq 2^{n}, \quad m \leq n, \quad \omega \in \Omega \backslash N^{n} \\
& \Rightarrow\left|\sum_{\left.\left.t \in \in^{*}\right] k^{-} 2^{-m}, k 2^{-m}\right] \cap T^{2}} \delta \alpha^{m}(\omega, t)-\sum_{\left.t \in \epsilon^{*}\right] k^{-} 2^{-m}, k 2^{-m} \mathrm{n} \cap \mathrm{T}^{2}} \delta \alpha^{n}(\omega, t)\right|<\frac{1}{n}, \\
& \sum_{t \in \mathbf{T}^{2}} \delta \alpha^{n}(\omega, t)=1, \quad \forall \omega \in \Omega .
\end{aligned}
$$


Using the Countable Comprehension Principle, we can extend the sequence $\left(\delta \alpha^{n}, N^{n}\right)_{n \in \mathbb{N}}$ to an internal sequence $\left(\delta \alpha^{n}, N^{n}\right)_{n \in *^{*} \mathbb{N}}$. Set

$$
C=\left\{n \in{ }^{*} \mathbb{N}:(5.5),(5.6),(5.7) \text { and (5.8) hold, and } 2^{n+1} \leq n_{0}\right\} \text {. }
$$

By the Internal Definition Principle (see [HL, Theorem 6.4]), $C$ is an internal set, which contains the (external) set $\mathbb{N}$. Hence there is $m \in C \backslash \mathbb{N}$.

We set $\delta \alpha=\delta \alpha^{\underline{m}}$. Observe that (c) is satisfied by (5.8), and (a) holds by (5.5) with $h=2^{\underline{m}+1}$. Set $N=\tilde{N} \cup N^{\underline{m}}$. Then $P(N)=0$ by (5.6), and for all $m \in \mathbb{N}$ and $0 \leq k_{1}, k_{2} \leq 2^{m},(5.7)$ implies that

$$
\begin{aligned}
\omega \in \Omega \backslash N & \left.\left.\Rightarrow \mid \sum_{\left.\left.t \in \in^{*}\right] k^{-} 2^{-m}, k 2^{-m}\right] \cap T^{2}} \delta \alpha^{m}(\omega, t)-\bar{\alpha}\left(\omega,{ }^{*}\right] k^{-} 2^{-m}, k 2^{-m}\right] \cap \mathbb{T}^{2}\right) \mid \\
& <\frac{1}{\underline{m}} .
\end{aligned}
$$

By (5.1) and (5.4), this implies that for all $m \in \mathbb{N}$ and $0 \leq k_{1}, k_{2} \leq 2^{m}$,

$$
\begin{aligned}
\left.\alpha\left(\omega,{ }^{*}\right] k^{-} 2^{-m}, k 2^{-m}\right] \cap{T^{2}}^{2} & \left.\left.=\operatorname{st}\left(\bar{\alpha}\left(\omega,{ }^{*}\right] k^{-} 2^{-m}, k 2^{-m}\right] \cap T^{2}\right)\right) \\
& =\operatorname{st}\left(\sum_{\left.\left.t \in \in^{*}\right] k^{-} 2^{-m}, k 2^{-m}\right] \cap \mathbf{T}^{2}} \delta \alpha^{m}(\omega, t)\right) \\
& =\Delta_{\left.\left.2^{-m}\right] k^{-}, k\right]} A(\omega) .
\end{aligned}
$$

This proves (b), and concludes the proof.

5.3. Corollary. Let $\left(A_{t}\right)_{t \in[0,1]^{2}}$ be a randomized stopping point, and let $\delta \alpha$ be the internal weight process and $N$ the null set given by Theorem 5.2. For any Borel set $B \subset[0,1]^{2}$,

(a) $\mathrm{st}^{-1}(B) \cap \mathrm{T}^{2}$ is a Borel subset of $\mathrm{T}^{2}$;

(b) $\int_{B} d_{t} A_{t}(\omega)=\alpha\left(\omega, \mathrm{st}^{-1}(B) \cap \mathrm{T}^{2}\right), \forall \omega \in \Omega \backslash N$.

Proof. (a) is a consequence of Theorem (2.2.6) of [SB]. Furthermore, by a classical Monotone Class argument, it is sufficient to prove (b) when $B$ is a rectangle with dyadic edges, $B=] s, t\left[, s<t, s, t \in \mathbf{D}^{2}\right.$. We fix $\omega \in \Omega \backslash N$, and only consider the case $s \ll t$.

Let $\mu_{\omega}$ be the random measure on $[0,1]^{2}$ whose distribution function is $t \mapsto A_{t}(\omega)$. By Theorem 5.2(b),

$$
\left.\left.\left.\left.\mu_{\omega}(] s, t\right]\right)=\alpha\left(\omega,{ }^{*}\right] s, t\right] \cap T^{2}\right) .
$$

The remainder of the proof follows that of Lemma (2.3.2) of [SB]. Since

$$
\left.\left.\mathrm{st}^{-1}(] s, t[) \subset^{*}\right] s, t\right] \subset \mathrm{st}^{-1}([s, t]),
$$

we get by (5.9) that

$$
\left.\left.\alpha\left(\omega, \mathrm{st}^{-1}(] s, t[) \cap \mathbf{T}^{2}\right) \leq \mu_{\omega}(] s, t\right]\right) \leq \alpha\left(\omega, \mathrm{st}^{-1}([s, t]) \cap \mathbf{T}^{2}\right) .
$$


Now

$$
\begin{aligned}
\alpha(\omega, & \left.\mathrm{st}^{-1}(] s, t[) \cap \mathbf{T}^{2}\right) \\
& =\lim _{n \rightarrow \infty} \alpha\left(\omega, \mathrm{st}^{-1}([s+(1 / n, 1 / n), t-(1 / n, 1 / n)]) \cap \mathbf{T}^{2}\right) \\
& =\mu_{\omega}(] s, t[)
\end{aligned}
$$

since

$$
\begin{aligned}
\mu_{\omega}(] s, t[) & \left.\left.=\lim _{n \rightarrow \infty} \mu_{\omega}(] s+(1 / n, 1 / n), t-(1 / n, 1 / n)\right]\right) \\
& \leq \lim _{n \rightarrow \infty} \alpha\left(\omega, \mathrm{st}^{-1}([s+(1 / n, 1 / n), t-(1 / n, 1 / n)]) \cap \mathbf{T}^{2}\right) \\
& \leq \lim _{n \rightarrow \infty} \alpha\left(\omega, \mathrm{st}^{-1}(] s+(1 / 2 n, 1 / 2 n), t-(1 / 2 n, 1 / 2 n)[) \cap \mathbf{T}^{2}\right) \\
& \left.\left.\leq \lim _{n \rightarrow \infty} \mu_{\omega}(] s+(1 / 2 n, 1 / 2 n), t-(1 / 2 n, 1 / 2 n)\right]\right) \\
& =\mu_{\omega}(] s, t[) .
\end{aligned}
$$

This completes the proof.

Theorem 5.2 and Corollary 5.3 provide the desired liftings of randomized stopping points. The projection theorem is simpler.

5.4. Projection Theorem. Let $\delta \alpha: \Omega \times \mathbf{T}^{2} \rightarrow^{*}[0,1]$ be an internal weight process, such that

$$
\sum_{t \in \mathrm{T}^{2}} \delta \alpha(\omega, t)=1, \quad \forall \omega \in \Omega \backslash M,
$$

where $M$ is an (internal) $\bar{P}$-null set. Suppose that for some $h \in{ }^{*} \mathbf{N} \backslash \mathbf{N}, \delta \alpha$ is adapted to the internal fibration $\left(\mathscr{A}_{t}^{h}\right)_{t \in \mathrm{T}^{2}}$, where $\mathscr{A}_{t}^{h}=\mathscr{A}_{t+(1 / h, 1 / h)}, \forall t \in \mathbf{T}^{2}$. Set

$$
\begin{gathered}
A_{t}(\omega)=\inf _{\left.\left.q \in \mathbf{D}^{2} \cap\right] t,(1,1)\right]} \alpha\left(\omega,{ }^{*}[0, q] \cap \mathrm{T}^{2}\right), \quad t \in[0,1]^{2} \backslash\{(1,1\}, \\
A_{(1,1)}(\omega) \equiv 1 .
\end{gathered}
$$

Then $A=\left(A_{t}\right)_{t \in[0,1]^{2}}$ is a randomized stopping point such that for almost all $\omega \in \Omega$,

$$
\int_{B} d_{t} A_{t}(\omega)=\alpha\left(\omega, \mathbf{s t}^{-1}(B) \cap \mathbf{T}^{2}\right),
$$

for all Borel sets $B \subset[0,1]^{2} \quad(A$ is termed the projection of $\delta \alpha)$.

Proof. The definition of $A$ clearly implies that $A .(\omega)$ is right-continuous and has positive planar increments. Since $A_{(1,1)} \equiv 1$ a.s., $A$ will be a randomized stopping point provided $A_{t}$ is $\mathscr{F}_{t}$-measurable, for all $t \in[0,1]^{2}$. This is the case since $\left(\mathscr{F}_{t}\right)$ is right-continuous and $\alpha\left(\cdot,{ }^{*}[0, q] \cap \mathrm{T}^{2}\right)$ is $\mathscr{F}_{q}$-measurable by Lemma 3.1. As for the last statement of the theorem, it is sufficient to observe that by the definition of $A, A_{q}(\omega)=\alpha\left(\omega, \mathbf{s t}^{-1}([0, q]) \cap \mathrm{T}^{2}\right), \forall q \in \mathbf{D}^{2}$. 


\section{EXTREMAL ELEMENTS OF THE SET OF RANDOMIZED STOPPING POINTS}

The purpose of this section is to show that on any filtered Loeb probability space that satisfies properties $F 1$ to $F 4$, all extremal elements of the set of randomized stopping points are (ordinary) stopping points. As mentioned in $\S 2$, this will be the key step in our proof of existence of optimal stopping points.

Throughout this section, we work, under Assumption 4.1, with a fixed randomized stopping point $A=\left(A_{t}\right)_{t \in[0,1]^{2}}$. Using the Lifting Theorem 5.2, together with the Splitting Theorem 2.2 and the Transfer Principle (3.3), we shall build two randomized stopping points $A^{i}=\left(A_{t}^{i}\right)_{t \in[0,1]^{2}}, i=1,2$, and an optional increasing path $Z^{*}$ such that

$$
A=\frac{1}{2} A^{1}+\frac{1}{2} A^{2}
$$

and $Z^{*}$ splits $[0,1]^{2}$ into two parts, one of which contains the support of the random probability measure associated with $A^{1}$, and the other, the support of the random measure associated with $A^{2}$ (of course, if $A$ is a stopping point, the supports of $A, A^{1}$ and $A^{2}$ will be contained in the graph of $Z^{*}$ ).

Let $\delta \alpha$ be the internal weight process given by Theorem 5.2, together with $h \in{ }^{*} \mathbb{N} \backslash \mathbb{N}$ and the $P$-null set $N: \delta \alpha$ is adapted to the internal (complete) filtration $\left(\mathscr{A}_{t}^{h}\right)_{t \in \mathrm{T}^{2}}$, which satisfies Hypothesis $\overline{\mathrm{F} 4}$.

Let $\mathbf{T}+\mathbf{T}=\{0, \Delta u, 2 \Delta u, \ldots, 2\}$. The Transfer Principle, applied to Theorem 2.2 in the case of a finite index set, affirms the existence of an internal $\bar{P}$-null set $M$, an internal function $\tilde{Z}: \Omega \times(T+T) \rightarrow T^{2}$ and of two internal weight processes $\delta \alpha^{1}, \delta \alpha^{2}: \Omega \times \mathrm{T}^{2} \rightarrow^{*}[0,1]$ with the following properties for all $t \in \mathbb{T}^{2}, \omega \in \Omega \backslash M, p \in \mathbb{T}+\mathrm{T}$ :

$$
\begin{gathered}
\delta \alpha(\omega, t)=\frac{1}{2} \delta \alpha^{1}(\omega, t)+\frac{1}{2} \delta \alpha^{2}(\omega, t) \\
\delta \alpha^{i} \text { is } \mathscr{A}_{t}^{h} \text {-measurable, } \quad i=1,2 ; \\
\sum_{s \in \mathbf{T}^{2}} \delta \alpha^{i}(\omega, s)=1, \quad i=1,2 ; \\
\tilde{Z}(\omega, p+\Delta u) \in\{\tilde{Z}(\omega, p)+(\Delta u, 0), \tilde{Z}(\omega, p)+(0, \Delta u)\} \\
\{\omega \in \Omega: \widetilde{Z}(\omega, p) \leq t\} \in \mathscr{A}_{t}^{h} ; \\
t \wedge \tilde{Z}(\omega,|t|) \Rightarrow\left(\delta \alpha^{1}(\omega, t)=2 \delta \alpha(\omega, t), \delta \alpha^{2}(\omega, t)=0\right) ; \\
\tilde{Z}(\omega,|t|) \wedge t \Rightarrow\left(\delta \alpha^{1}(\omega, t)=0, \delta \alpha^{2}(\omega, t)=2 \delta \alpha(\omega, t)\right)
\end{gathered}
$$

Let $A^{i}$ be the projection of $\delta \alpha^{i}, i=1,2$. It follows from the definition of $A^{i}$ (see Theorem 5.4) and from (6.2) that (6.1) holds. It remains to be shown that if $A$ is not in fact a stopping point, then $A^{1} \neq A \neq A^{2}$.

Recall that a map $f: \mathbf{T} \rightarrow \mathbf{T}^{2}$ is termed $S$-continuous provided $u \approx v \Rightarrow$ $f(u) \approx f(v), \forall u, v \in \mathrm{T}$ (see [SB, Appendix 1.4]). 
6.1. Lemma. (a) For $\omega \in \Omega \backslash M, p \mapsto \widetilde{Z}(\omega, p)$ is $S$-continuous;

(b) Define $Z^{*}=\left(Z_{u}^{*}\right)_{u \in[0,2]}$ by $Z_{u}^{*}(\omega)=\operatorname{st}\left(\tilde{Z}\left(\omega, \mathrm{st}^{-1}(u)\right)\right), \omega \in \Omega, u \in$ $[0,2]$. Then $Z$ is an optional increasing path.

Proof. Property (a) is a consequence of the equality

$$
|\tilde{Z}(\omega, p)-\tilde{Z}(\omega, q)|=|p-q|, \quad \forall \omega \in \Omega, p, q \in \mathbf{T}+\mathbf{T},
$$

which follows from (6.5). As for (b), observe that $Z_{u}^{*}$ is well defined by (a), since if $\omega$ is not in the $\bar{P}$-null set $M$ and $u=\operatorname{st}(p)=\operatorname{st}(\tilde{p})$, then $\widetilde{Z}(\omega, p) \approx$ $\widetilde{Z}(\omega, \tilde{p})$, so $\operatorname{st}(\widetilde{Z}(\omega, p))=\operatorname{st}(\widetilde{Z}(\omega, \tilde{p}))$. Furthermore, $u \mapsto Z_{u}^{*}(\cdot)$ is increasing by (6.5), and if $p \in \mathbf{T}+\mathbf{T}$ is such that $\operatorname{st}(p)=u$, then

$$
\left|Z_{u}^{*}(\omega)\right|=\operatorname{st}(|\tilde{Z}(\omega, p)|)=\operatorname{st}(p)=u,
$$

also by (6.5). Now fix $u \in[0,2]$ and $t \in[0,1]^{2}$. We must show that

$$
\left\{\omega \in \Omega: Z_{u}^{*}(\omega) \leq t\right\} \in \mathscr{F}_{t} .
$$

Since the filtration $\left(\mathscr{F}_{t}\right)$ is right-continuous, it is sufficient to show that for $t \in[0,1]^{2} \cap \mathbf{D}^{2}$,

$$
F=\{\omega \in \Omega: \operatorname{st}(\tilde{Z}(\omega, p)) \ll t\} \in \mathscr{F},
$$

where $p \in \mathbf{T}+\mathbf{T}$ is such that $p \approx u$. Since $t$ also belongs to $\mathbf{T}^{2}$,

$$
F=\bigcup_{n \in \mathbf{N}}\{\omega \in \Omega: \tilde{Z}(\omega, p) \leq t-(1 / n, 1 / n)\} .
$$

But then (6.6) implies that $F \in \mathscr{F}_{t}$. This completes the proof.

The following lemma shows that $A^{1}, A^{2}$ and $Z^{*}$ have a property similar to that of Theorem 2.2(b2).

6.2. Lemma. Fix $\omega \in \Omega \backslash M$ and $s, t \in \mathrm{D}^{2}$ such that $s \leq t$.

(a) Suppose $\left(t_{1}, s_{2}\right) \wedge Z_{t_{1}+s_{2}}^{*}(\omega)$. Then $\Delta_{\mathrm{ls}, t]} A^{2}(\omega)=0$.

(b) Suppose $Z_{s_{1}+t_{2}}^{*}(\omega) \wedge\left(s_{1}, t_{2}\right)$. Then $\Delta_{\mathrm{ls}, t]} A^{1}(\omega)=0$.

Proof. We only prove (a). By the hypothesis and (6.7), there is $\varepsilon>0, \varepsilon \in \mathbf{R}$, such that

$$
\left.\left.\delta \alpha^{2}(\omega, u)=0, \quad \forall u \in \in^{*}\right] s, t+(\varepsilon, \varepsilon)\right] \cap \mathbf{T}^{2},
$$

so if $\left.\left.p, q \epsilon^{*}\right] s, t+(\varepsilon, \varepsilon)\right] \cap \mathbf{D}^{2}, p \leq q$,

$$
\sum_{p<u \leq q} \delta \alpha^{2}(\omega, u)=0
$$

Thus, where $\alpha^{2}(\omega,[a, b])$ is an abbreviation of $\alpha^{2}\left(\omega,{ }^{*}[a, b] \cap \mathbf{T}^{2}\right)$,

$$
\alpha^{2}(\omega,[0, q])-\alpha^{2}\left(\omega,\left[0,\left(p_{1}, q_{2}\right)\right]\right)-\alpha^{2}\left(\omega,\left[0,\left(q_{1}, p_{2}\right)\right]\right)+\alpha^{2}(\omega,[0, p])=0 \text {. }
$$

Taking the limit as $q \downarrow t, p \downarrow s$ gives the desired result.

If $\left(Z_{u}\right)_{u \in[0,2]}$ is an o.i.p. we set $\operatorname{Im} Z .(\omega)=\left\{Z_{u}(\omega): 0 \leq u \leq 2\right\}$, and if $\nu$ is a measure, $\operatorname{supp} \nu$ denotes the support of $\nu$. 
6.3. Proposition. Suppose $\mu_{\omega}(\cdot)$ is the random measure whose distribution function is the randomized stopping point $\left(A_{t}\right)_{t \in[0,1]^{2}}$, and suppose

$$
P\left\{\omega \in \Omega: \operatorname{supp} \mu_{\omega}(\cdot) \subset \operatorname{Im} Z .(\omega)\right\}<1
$$

for all o.i.p.'s $\left(Z_{u}\right)_{u \in[0,2]}$. Then $A^{1} \neq A \neq A^{2}$.

Proof. Let $\left(Z_{u}^{*}\right)_{u \in[0,2]}$ be the o.i.p. defined in Lemma 6.1, and set

$$
F^{*}=\left\{\omega \in \Omega: \operatorname{supp} \mu_{\omega}(\cdot) \not \subset \operatorname{Im} Z_{.}^{*}(\omega)\right\} .
$$

Since $P\left(F^{*}\right)>0$, we may suppose for example that $P(F)>0$, where $F=\left\{\omega \in \Omega:\right.$ there is $s, t \in \mathbb{D}^{2}, s \leq t$ such that $\left(t_{1}, s_{2}\right) \wedge Z_{t_{1}+s_{2}}^{*}(\omega)$

$$
\text { and } \left.\Delta_{\mathrm{j}, t]} A(\omega)>0\right\} \text {. }
$$

Now for each $\omega \in F \backslash M$, since $A=\frac{1}{2} A^{1}+\frac{1}{2} A^{2}$, we have by Lemma 6.2:

$$
\Delta_{\mathrm{\jmath} s, t]} A^{1}(\omega)=2 \Delta_{\mathrm{j} s, t]} A(\omega) \neq \Delta_{\mathrm{\jmath} s, t]} A(\omega)
$$

for some $s, t \in \mathbb{D}^{2}$ with $s \leq t$. This implies that the sample paths $t \mapsto A_{t}(\omega)$, $t \mapsto A_{t}^{i}(\omega), i=1,2$, are distinct for $\omega \in F \backslash M$. Since $P(F \backslash M)>0$, $A^{1} \neq A \neq A^{2}$.

The following lemma is a straightforward extension of a result for singleparameter randomized stopping points.

6.4. Lemma. Let $(\Omega, \mathscr{F}, P)$ be an arbitrary (complete) probability space, and $\left(\mathscr{F}_{t}\right)_{t \in[0,1]^{2}}$ an arbitrary two-parameter filtration (with or without CQI or F4). Suppose $\mu_{\omega}(\cdot)$ is a random measure whose distribution function is some randomized stopping point $A=\left(A_{t}\right)_{t \in[0,1]^{2}}$. If there is an optional increasing path $\left(Z_{u}\right)_{u \in[0,2]}$ such that

$$
P\left\{\omega \in \Omega: \operatorname{supp} \mu_{\omega}(\cdot) \subset \operatorname{Im} Z .(\omega)\right\}=1,
$$

then $A$ is an extremal element of the set of randomized stopping points if and only if $A$ is a stopping point.

Proof. Set $B_{t}^{1}=\min \left(2 A_{t}, 1\right), B_{t}^{2}=\max \left(2 A_{t}-1,0\right)$. Clearly $A_{t}=\frac{1}{2} B_{t}^{1}+\frac{1}{2} B_{t}^{2}$, and the sample paths

$$
t \mapsto A_{t}(\omega) \text { and } t \mapsto B_{t}^{i}(\omega), \quad i=1,2,
$$

are distinct if and only if $0<A_{t}(\omega)<1$ for some $t$. If $s, t \in[0,1]^{2}$ are such that $s \leq t$, it is easy to see that $\Delta_{\mathrm{l} s, t]} B^{i} \geq 0$ a.s. by examining the relative positions of $s, t$ and the path $u \mapsto Z_{u}$ (see Figure 1).

Since $B_{(1,1)}^{i} \equiv 1$, this implies that $B^{1}$ and $B^{2}$ are randomized stopping points. Thus if $A$ is extremal, we must have

$$
A_{t} \in\{0,1\} \text { a.s. }
$$

But then $A$ is a stopping point.

It is now straightforward to prove the continuous time extension of Theorem 2.2 . 


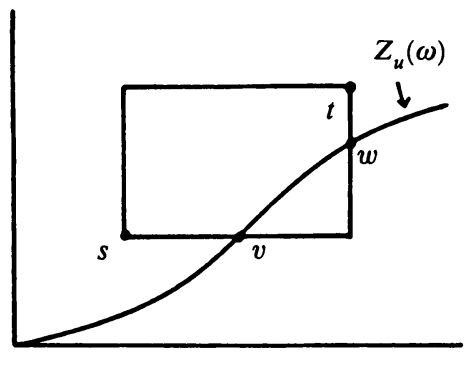

$\Delta_{\mathrm{ls}, t]} B^{i}(\omega)=B_{w}^{i}(\omega)-B_{v}^{i}(\omega) \geq 0$

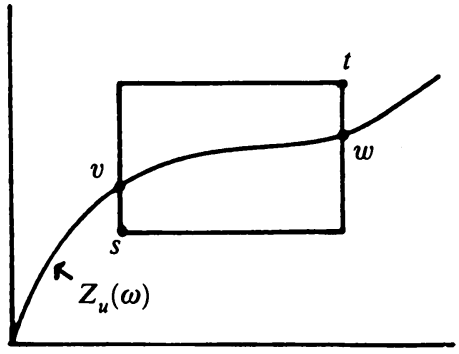

$\Delta_{\mathrm{l}, t]} B^{i}(\omega)=B_{w}^{i}(\omega)-B_{v}^{i}(\omega) \geq 0$

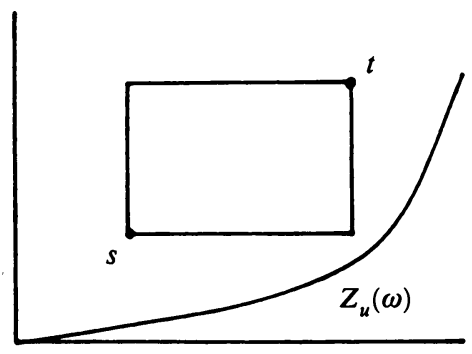

$\Delta_{\mathrm{ls}, t]} B^{i}(\omega)=0$

FIGURE 1

6.5. Theorem. Let $(\Omega, \mathscr{A}, \bar{P})$ be an internal probability space, $(\Omega, L(\mathscr{A}), P)$ the corresponding Loeb space. Suppose $\left(\mathscr{F}_{t}\right)_{t \in[0,1]^{2}}$ is the standard part of an internal (complete) two-parameter filtration that satisfies Hypothesis $\overline{\mathrm{F} 4}$. Then all extremal elements of the set of randomized stopping points are stopping points.

Proof. Let $A=\left(A_{t}\right)_{t \in[0,1]^{2}}$ be a randomized stopping point. Suppose

$$
P\left\{\omega \in \Omega: \operatorname{supp} \mu_{\omega}(\cdot) \subset \operatorname{Im} Z .(\omega)\right\}<1
$$

for all optional increasing paths $Z$, where $\mu_{\omega}(\cdot)$ is the measure on $[0,1]^{2}$ whose distribution function is $t \mapsto A_{t}(\omega)$. Then by Proposition 6.3, $A$ is the midpoint of two distinct randomized stopping points, and thus is not extremal. This implies that any extremal randomized stopping point must satisfy

$$
P\left\{\omega \in \Omega: \operatorname{supp} \mu_{\omega}(\cdot) \subset \operatorname{Im} Z .(\omega)\right\}=1
$$

for some optional increasing path $Z$. But then the statement of the theorem is a consequence of Lemma 6.4 . 
6.6. Remark. It is not known whether the conclusion of this theorem remains valid for filtered probability spaces that satisfy Hypothesis F4 but are not Loeb spaces.

\section{ApPliCATION: The EXISTENCE OF OPTIMAL STOPPING POINTS}

As mentioned in $\S 2$, Theorem 6.5 leads to a proof of the existence of optimal stopping points, under integrability assumptions as weak as those in [K], Theorem 1]. For this we need the following proposition.

7.1. Proposition. Let $(\Omega, \mathscr{F}, P)$ be an arbitrary complete probability space, and $X=\left(X_{t}\right)_{t \in[0,1]^{2}}$ a measurable process with upper semicontinuous (u.s.c.) sample paths such that $E\left(\sup _{t \in[0,1]^{2}} X_{t}\right)<+\infty$. Then the map $\Phi_{X}: \mathscr{U} \rightarrow \mathbb{R} \cup\{-\infty\}$ defined by

$$
\Phi_{X}\left(\left(A_{t}\right)_{t \in[0,1]^{2}}\right)=E\left(\int_{[0,1]^{2}} X_{t}(\cdot) d_{t} A_{t}(\cdot)\right)
$$

is u.s.c. (for the weak topology induced by $\sigma\left(\mathscr{C}^{*}, \mathscr{C}\right)$; see $\S 2$ ).

Proof. For separable bounded processes, this was proved in [D1, Theorem 3.5]. Our proof here is more direct and gives the more general result above.

We should perhaps point out that the map $\omega \mapsto \sup _{t \in[0,1]^{2}} X_{t}$ is measurable since the process $X$ is (see the proof of [DM, IV. 33a]), and so it makes sense to speak of sup integrability for $X$, and $\Phi_{X}$ is well-defined.

If the sample paths of the process $X$ were continuous, then the function $\Phi_{X}$ would be continuous by the definition of the weak topology $\sigma\left(\mathscr{C}^{*}, \mathscr{C}\right)$. Now suppose there were a nonincreasing sequence $\left(Y^{k}\right)_{k \in N}$ of continuous processes in $\mathscr{C}$ such that

$$
\lim _{k \rightarrow \infty} \downarrow Y_{t}^{k}(\omega)=X_{t}(\omega),
$$

for almost all $\omega \in \Omega$. Then we would have $\Phi_{Y^{k}} \downarrow \Phi_{X}$ by monotone convergence, and so $\Phi_{X}$, as the nonincreasing limit of a sequence of continuous functions, would be u.s.c. [B1, IV.6.2 Theorem 4]. Thus the proposition will be proved if we construct the sequence $\left(Y^{k}\right)_{k \in N}$.

It is well known that an u.s.c. bounded function defined on a metric space is the nonincreasing limit of a sequence of continuous functions, so the problem here is to choose the sequence for fixed $\omega \in \Omega$ in such a way that the resulting $Y_{t}^{k}(\omega)$ are measurable functions of $\omega$ and such that $Y^{k} \in \mathscr{C}$. In order to do this, we need the following lemma.

7.2. Lemma. Consider $F \subset \mathscr{F} \times \mathscr{B}\left(\bar{I}^{n}\right)$ such that for each $\omega \in \Omega$, the section $F_{\omega}=\left\{t \in \bar{I}^{n}:(\omega, t) \in F\right\}$ is closed. Then the mapping $\omega \mapsto \operatorname{dist}\left(t, F_{\omega}\right)$ is $\mathscr{F}$-measurable $\left(\operatorname{dist}\left(t, F_{\omega}\right)\right.$ denotes the distance between $t$ and the set $F_{\omega}$ for the usual metric on $\bar{I}^{n}$ ).

Proof. For $r>0$,

$$
A=\left\{\omega \in \Omega: \operatorname{dist}\left(t, F_{\omega}\right)<r\right\}=\left\{\omega \in \Omega: \text { there is } s \in F_{\omega}, d(s, t)<r\right\},
$$


so $A$ is the projection on $\Omega$ of the $\mathscr{F} \times \mathscr{B}\left(\bar{I}^{n}\right)$-measurable set $F \cap(\Omega \times B(t, r))$, where $B(t, r)$ denotes the open ball centered at $t$ with radius $r$. Thus $A$ is $\mathscr{F}$-analytic by Theorem II.13 of [DM], and since $\mathscr{F}$ is complete, $F \in \mathscr{F}$ by III.33 of [DM]. This proves the lemma.

End of the proof of Proposition 7.1. Our proof follows that of [B1, IX $\S 2.7$, Proposition 11]. Since we can always replace the process $X$ by the process $\left(X_{t}-\sup _{t} X_{t}\right)_{t \in[0,1]^{2}}$, we may suppose without loss of generality that $X \leq 0$. Set

$$
X_{t}^{n}(\omega)=-2^{-n} \sum_{k=1}^{\infty} I_{U^{k, n}}(\omega, t)
$$

where

$$
U^{k, n}=\left\{(\omega, t) \in \Omega \times \bar{I}^{n}: X_{t}(\omega)<-k 2^{-n}\right\},
$$

and observe that $\left(X^{n}\right)_{n \in N}$ is a nonincreasing sequence which converges to $X$. Now since $X$ is u.s.c., the section $U_{\omega}^{k, n}$ of $U^{k, n}$ is open for each $\omega \in \Omega$. Furthermore, since $\sup _{t} X_{t}<+\infty$ a.s., there is a measurable map $\omega \mapsto K_{\omega}$ from $\Omega$ into $\mathrm{N}$ such that $k>2^{n} K_{\omega} \Rightarrow I_{U^{k, n}}(\omega, t)=0, \forall t$, for almost all $\omega \in \Omega$.

For each fixed $k, l$, and $n$, set

$$
Z_{t}^{k, n, l}(\omega)=\min \left(1, l \operatorname{dist}\left(t, \bar{I}^{n} \backslash U_{\omega}^{k, n}\right)\right) .
$$

Then $\omega \mapsto Z^{k, n, l}(\omega)$ is a measurable map by Lemma 7.2, $t \mapsto Z_{t}^{k, n, l}(\omega)$ is continuous and

$$
\left(t \in \bar{I}^{n} \backslash U_{\omega}^{k, n} \text { or } \operatorname{dist}\left(t, \bar{I}^{n} \backslash U^{k, n}\right)>1 / l\right) \Rightarrow Z_{t}^{k, n, l}(\omega)=I_{U^{k, n}}(\omega, t),
$$

so

$$
\lim _{l \rightarrow \infty} \uparrow Z_{t}^{k, n, l}(\omega)=I_{U^{k, n}}(\omega, t), \quad \forall t \in \bar{I}^{n}, \quad \forall \omega \in \Omega .
$$

Thus if we define a bounded continuous process $X^{n, l}$ by setting

$$
X_{t}^{n, l}(\omega)=\max \left(-2^{-n} \sum_{k=1}^{2^{n} K_{\omega}} Z_{t}^{n, k, l}(\omega)-l\right),
$$

we have

$$
\lim _{l \rightarrow \infty} \downarrow X_{t}^{n, l}(\omega)=X_{t}^{n}(\omega), \quad \forall t \in \bar{I}^{n}, \quad \text { for almost all } \omega \in \Omega .
$$

But then the sequence $\left(Y^{k}\right)_{k \in N}$ of continuous processes defined by

$$
Y_{t}^{k}(\omega)=\min _{n, l \leq k} X_{t}^{n, l}(\omega)
$$

satisfies the required conditions.

7.2. Theorem. Let $\left(\Omega, \mathscr{F}, P,(\mathscr{F})_{t \in[0,1]^{2}}\right)$ be a filtered Loeb space satisfying the assumptions of Theorem 6.5, and let $\left(X_{t}\right)_{t \in[0,1]^{2}}$ be a measurable process with 
upper semicontinuous sample paths, such that $E\left(\sup _{t \in[0,1]^{2}} X_{t}\right)<+\infty$. Then there is a stopping point $T_{0}$ such that

$$
E\left(X_{T_{0}}\right)=\sup _{T \in \mathscr{T}} E\left(X_{T}\right)
$$

Proof. This proof is similar to that of Ghoussoub [G, Proposition II.3]. Consider the functional $\Phi: \mathscr{U} \rightarrow \mathbf{R}$ defined by

$$
\Phi\left(\left(A_{t}\right)_{t \in[0,1]^{2}}\right)=E\left(\int_{[0,1]^{2}} X_{t}(\cdot) d_{t} A_{t}(\cdot)\right) .
$$

By Lemma 7.1, this functional is u.s.c. on $\mathscr{U}$. Since $\Phi$ is affine, it attains its maximum on $\mathscr{U}$ at an extremal element $A^{0} \in \operatorname{ext} \mathscr{U}$ [B2, II $\S 7$, Proposition 1]. By Theorem $6.5, A^{0}$ is in fact a stopping point, which we denote $T_{0}$. This stopping point is clearly optimal.

7.3. Remark. From the point of view of applications, it does not seem too restrictive to impose that the underlying probability space be Loeb. In the singleparameter case, this would be no restriction at all due to the result of Hoover and Keisler [HK], which shows that these spaces are universal and saturated.

7.4. Remark. The papers [ $\mathrm{Mi}$ and $\mathrm{MM}$ ] claim, under certain regularity assumptions on the reward process, the existence of optimal stopping points in the two-parameter optimal stopping problem on arbitrary probability spaces (in [MM], there is even no Hypothesis F4 on the filtration). Both these papers use a "randomized" set $\mathscr{U}$ which is different from the one considered here, and both papers use the following theorem: "a separately continuous bilinear map is jointly continuous", in a situation where the hypothesis of this theorem is not satisfied [Mi, Theorem 1.5; MM, Proposition 7]. Thus the problem of existence of optimal stopping points on arbitrary probability spaces, even for continuous processes and under Hypothesis F4, is open.

Acknowledgment. The author thanks E. Perkins for pointing out that the initial proof for hyperfinite probability spaces carried over to all Loeb spaces, and M. J. Klass for bringing the results of $[\mathrm{Kl}]$ to his attention.

\section{REFERENCES}

[AFHL] S. Albeverio, J. E. Fenstad, R. Høegh-Kron and T. Lindstrøm, Nonstandard methods in stochastic analysis and mathematical physics, Academic Press, New York, 1986.

[A] R. Anderson, A non-standard representation of Brownian motion and Itô integration, Israel J. Math. 25 (1976), 15-46.

[Ba] M. T. Barlow, One dimensional stochastic differential equations with no strong solution, J. London Math. Soc. (2) 23 (1982), 335-347.

[BC] J. R. Baxter and R. V. Chacon, Compactness of stopping times, Z. Wahrsch Verw. Gebiete 40 (1977), 169-181.

[B1] N. Bourbaki, Eléments de mathématique topologie générale, Chaps. I-IV, Hermann, Paris, 1971, and Chaps. V-X, Hermann, Paris, 1974.

[B2] __ Eléments de mathématique espaces vectoriels topologiques, Chaps. I-V, Hermann, Paris, 1981. 
[CG] R. Cairoli and J. P. Gabriel, Arrêt optimal de certaines suites de variables aléatoires indépendantes, Séminaire de Probabilité XIII, Lecture Notes in Math., vol. 721, Springer-Verlag, Berlin, Heidelberg and New York, 1978, pp. 174-198.

[CW] R. Cairoli and J. B. Walsh, Stochastic integrals in the plane, Acta Math. 134 (1975), 11-183.

[D1] R. C. Dalang, Sur larrêt optimal de processus à temps multidimensionnel continu, Séminaire de Probabilité XVIII, Lecture Notes in Math., vol. 1059, Springer-Verlag, Berlin, Heidelberg and New York, 1984, pp. 379-390.

[D2] __ On infinite perfect graphs and randomized stopping points on the plane, Probab. Theory and Related Fields 78 (1988), 357-378.

[DTW] R. C. Dalang, L. E. Trotter Jr., and D. de Werra, On randomized stopping points and perfect graphs, J. Combin. Theory Ser. B 45 (1988), 320-344.

[DM] C. Dellacherie and P. A. Meyer, Probabilités et potentiel, Chaps. I-IV, Hermann, Paris, 1975.

[G] N. Ghoussoub, An integral representation of randomized probabilities and its applications, Séminaire de Probabilité XVI, Lecture Notes in Math., vol. 920, Springer-Verlag, Berlin, Heidelberg and New York, 1982, pp. 519-543.

[HK] D. N. Hoover and H. J. Keisler, Adapted probability distributions, Trans. Amer. Math. Soc. 286 (1984), 159-201.

[HP] D. N. Hoover and E. Perkins, Nonstandard construction of the stochastic integral and applications to stochastic differential equations. I, II, Trans. Amer. Math. Soc. 275 (1983), 1-58.

[HL] A. E. Hurd and P. Loeb, An introduction to non-standard real analysis, Academic Press, New York, 1985.

[K] H. J. Keisler, Foundations of infinitesimal stochastic analysis, Mem. Amer. Math. Soc., vol. 48, no. 297 (1984).

[K1] M. J. Klass, Properties of optimal extended-valued stopping rules for $S_{n} / n$, Ann. Probab. 1 (1973), 719-757.

[KS] U. Krengel and L. Sucheston, Stopping rules and tactics for processes indexed by a directed set, J. Multivariate Anal. 11 (1981), 199-229.

[L] P. A. Loeb, Conversion from non-standard to standard measure spaces and applications in probability theory, Trans. Amer. Math. Soc. 211 (1975), 113-122.

[MV] A. Mandelbaum and R. J. Vanderbei, Optimal stopping and supermartingales over partially ordered sets, Z. Wahrsch. Verw. Gebiete 57 (1987), 153-264.

[MMe] L. Manewitz and E. Merzbach, Multi-parameter stochastic processes via non-standard analysis (preprint).

[MS] G. Mazziotto and J. Szpirglas, Arrêt optimal sur le plan, Z. Wahrsch. Verw. Gebiete 62 (1983), 215-233.

[Ma] G. Mazziotto, Two parameter optimal stopping and bi-Markov processes, Z. Wahrsch. Verw. Gebiete 69 (1985), 99-135.

[MM] G. Mazziotto and A. Millet, Points, liques et systèmes d'arrêt flous et problème d'arrêt optimal, Séminaire de Probabilité XX, Lecture Notes in Math., vol. 1204, Springer-Verlag, Berlin, Heidelberg and New York, 1986, pp. 81-94.

[Me] P. A. Meyer, Convergence faible et compacité des temps d'arrêt d'après Baxter et Chacon, Séminaire de Probabilité XII, Lecture Notes in Math., vol. 850, Springer-Verlag, Berlin, Heidelberg and New York, 1978, pp. 411-423.

[Mi] A. Millet, On randomized tactics and optimal stopping in the plane, Ann. Probab. 13 (1985), 946-965.

[SB] K. D. Stroyan and J. M. Bayod, Foundations of infinitesimal stochastic analysis, North-Holland, New York, 1986.

[W] J. B. Walsh, Optional increasing paths, Proc. Aléatoires à Deux Indices, Lecture Notes in Math., vol. 863, Springer-Verlag, Berlin, Heidelberg and New York, 1981, pp. 172-201.

Department of Statistics, University of California, Berkeley, California 94720 